\title{
Adaptive Improved PCA with Wavelet Transform for Image Denoising
}

\author{
Vikas Gupta \\ Technocrats Institute of technology \\ Bhopal, MP
}

\author{
Amruta V. Band \\ Technocrats Institute of technology \\ Bhopal, MP
}

\begin{abstract}
Removing Noise from the original image is yet a gainsaying problem for research workers. There have been various algorithms proposed for noise removal and each algorithm has its advantages, assumptions and drawbacks. In this paper image denoising problem can be solved by using combine approach of Principal component analysis and wavelet transform. Wavelet transform applied on image for contrast enhancement where as Principal component analysis is used for noise removal. The database outcomes of proposed algorithm show that proposed algorithm, improves the Peak signal noise ratio by denoising the image effectively and keeping the data of original image better.
\end{abstract}

\section{Keywords}

Discrete wavelet transforms (DWT); Peak signal to noise ratio (PSNR); Principal component analysis (PCA)

\section{INTRODUCTION}

Unwanted signals called noise will corrupt the image at the time of transmission or at the time of acquirement, to remove this unwanted signals image denoising is an only step to prevent the degradation of image and which amend the quality of the image. At the primary phase of image processing, noise suppression has been studied and number of noise removal techniques have been published, like from the very first filters and frequency domain noise removal techniques [14] to the recently developed wavelet [1-10], sparse representation [13], curvelet [7] ,bilateral filtering [17,18],ridgelet [8] based methods, and K-SVD [15] methods, shape-adaptive transform [16], etc With the speedy growth of advanced digital imaging devices and their progressively broad uses in our day to day life, raises the demand of modern denoising techniques for more eminent quality of image. The wavelet transform has proven to be an efficient noise removal technique [1-10]. Wavelet transform decayed the image into multiple scales, this scales presents different time frequency elements of the original image. At each Scale, various operations like statistical modeling, thresholding are performed to reduce noise. Though the efficiency of wavelet transforms in removing noise is good but it shows the image by utilizes a determined wavelet basis (with dilation and translation). But for natural images, representation by utilizing only one determined wavelet basis is not advantageous, because image can acquainted with many false visual features in denoising result. This problem was solved in [19] by finding a locally matched basis to translate the image.

Though removing noise from an image is a critical technology but it is basic and vivid research area in the image processing because of its underlying role in many applications. In this paper the problem of image denoising is investigated when Gaussian noise corrupts the image. Gaussian noise added to the image due to image acquisition. A bulk of work on image denoising is done in wavelet transform [10], and in Principal component analysis [12] which are effective and simple noise reduction methods.
In this paper, the ideas of discrete wavelet transform for improving contrast of image proposed by M. Prabukumar and J. Cristopher Clement [9] and adaptive principal components analysis [19] for noise removal proposed by D. Darian Muresan, Thomas W. Parks are used and making improvements by combining the advantages of these two methods. Denoising technique used here performs very well in case of PSNR values and gives very good result in case of visual fidelity of the image. The technique used here gives a good result as compare to other denoising methods like Gaussian Filter, median filter, Lee filter and Weiner filter.

The paper is organized as follows.. In Section 2, Details of PCA are discussed. The Section 3 presents the details of Discrete wavelet transform. Section 4 shows Computational Details. Section 5 presents Proposed algorithm. In section 6 experimental results are given and discussion while concluding remarks are given in Section 7.

\section{PRINCIPAL COMPONENT ANALYSIS}

Principal component analysis is also called as Karhunen Loeve transform. PCA is a hefty technique for data dimension reduction, as it is hard to get the shapes in data having large dimension where almost all graphical representation is inaccessible [12]. Reduction in dimension of data, sum up the data with a lower amount of variables, missing as less information as possible.PCA identifies data patterns and give their similarities and differences, it decomposes image pixel into locally adapted principal components, threshold the coefficients and reconstruct it.PCA also used in regression analysis. Naturally, the vitality of a signal will more in a minor subset of the PCA transform domain, on the other hand noise energy will distribute over the entire set of data evenly. Therefore, by keeping only the bulk of a crucial subset of the translated set of data and then taking the inverse PCA transform, the unwanted signal could be faithfully reduced and then the signal will be well recovered [20].

In this paper, PCA is used in two method Normal and Fast .In Fast method we are having adaptive Principal component's method, and in normal mode eight neighborhood method is there. The time required to obtain denoised image using the fast method is less as compared to normal method. For image denoising, PCA technique was suggested by utilizing a moving window to figure out the local statistics [12]. The optimal dimensionality suppression characteristic of PCA can also be utilized for removing noise.

\section{DISCRETE WAVELET TRANSFORM (DWT2)}

A novel algorithm for enhancing the contrast of images is proposed in [9] using DWT.

Representation of the image can be done in two ways using wavelet transforms that is in local spatial and frequency contains. The DCT and Fourier transform fail to give the local frequency property of the image. It only gives the global 
frequency property of an image. This limitation is eliminated by using scaling properties of wavelet transform [9].

Horizontal direction, vertical direction, approximate and detail coefficients can be used to decomposed signal.

In this paper approximate coefficient method used for contrast enhancement, which is given by

Where,

$$
\mathrm{CEI}=\frac{\mathrm{CP}+\mathrm{NP}}{2}
$$

$$
\begin{aligned}
\mathrm{CEI} & =\text { contrast enhance image } \\
\mathrm{CP} & =\text { current pixel } \\
\mathrm{NP} & =\text { next pixel }
\end{aligned}
$$

While using a color image, DWT must apply separately for each component that is on Red component, Green Component, and on Blue component. And for Gray color image DWT can apply once.

\section{COMPUTATIONAL TIME}

The calculation time is a crucial parameter in evaluating the functioning of all denoising techniques. The execution time for denoising a 256x256x3 RGB image by using Matlab R2011b on a Windows 7-based personal computer with i5 $2.50-\mathrm{GHz}$ CPU processor and 8 GB RAM is about 52.10s in normal mode and $46.47 \mathrm{~s}$ in fast mode and execution time for denoising a 256x256 grayscale image by using Matlab R2011b on a Windows 7-based personal computer with i5 $2.50-\mathrm{GHz} \mathrm{CPU}$ processor and $8 \mathrm{~GB}$ RAM is about $43.96 \mathrm{~s}$ in normal mode, and $8.53 \mathrm{~s}$ in fast mode

\section{ALGORITHM SUMMARY}

Following is the outline for proposed algorithm, which is named as IA-PCA(Improved adaptive-Principal component analysis) in this paper, and which is shown in Figure 1. Additive white Gaussian noise with noise level is assumed to be added in the image.

1) Take an Image

2) Choose method Fast or Normal

3) Employ Discrete wavelet Transform on the original image

4) Add Noise to image

5) Select automatic optimum window size

6) Select optimum neighborhood

7) Employ PCA on noisy image to remove noise

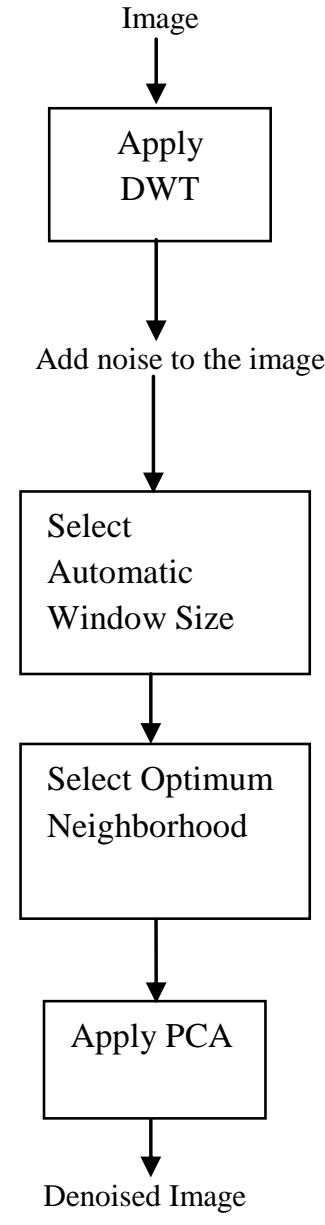

Fig.1 Flow chart of the proposed IA-PCA image denoising method

\section{RESULTS}

Using different images (Gray and RGB image), IA-PCA denoising method is examined against four different algorithms. Three images used here for tests are the standard images of Lenna, cameraman and monarch ,all are gray level. And another three RGB level images are Barbara, house and parrot. All images are tested by mixing noise with different noise level using randn MATLAB command.

Along with IA- PCA denoising, Gaussian, Weiner, Lee, Median are the four algorithms used for examining here.

The results for noise level 50, 20and 10, are listed out in Tables 2 , for gray level images and result of RGB color images are listed out in Tables 3. Comparison of different algorithm is given in Table 1 and almost in all tests the IA- PCA denoising method performed best in price of Peak signal noise ratio values. Looking at the denoising results for noise level $=20$, the comparison between the four denoising algorithms and proposed method are very noticeable. 
Table.1: Comparison of PSNR (in db) values with different denoising technique

\begin{tabular}{|c|c|c|c|c|c|c|}
\hline Image Name & Median & Lee & Weiner & Gaussian & \multicolumn{2}{|c|}{ Proposed } \\
\hline & & & & & Normal & Fast \\
\hline Lenna & 22.9481 & 25.7506 & 26.0118 & 26.3957 & 53.70 & 53.61 \\
\hline Cameraman & 20.8301 & 25.69 & 25.65 & 24.37 & 54.00 & 54.18 \\
\hline Monarch & 20.61 & 24.82 & 24.67 & 25.23 & 51.72 & 51.66 \\
\hline
\end{tabular}

Table.2: Comparison of PSNR-1 \& PSNR-2 values for gray images

\begin{tabular}{|c|c|c|c|c|c|c|c|c|c|c|c|c|}
\hline \multirow{3}{*}{$\begin{array}{l}\text { Noise } \\
\text { level }\end{array}$} & \multicolumn{4}{|c|}{ Leena.tif } & \multicolumn{4}{|c|}{ Cameraman.tif } & \multicolumn{4}{|c|}{ Monarch.tif } \\
\hline & \multicolumn{2}{|c|}{ Normal } & \multicolumn{2}{|c|}{ Fast } & \multicolumn{2}{|c|}{ Normal } & \multicolumn{2}{|c|}{ Fast } & \multicolumn{2}{|c|}{ Normal } & \multicolumn{2}{|c|}{ Fast } \\
\hline & $\begin{array}{c}\text { PSNR- } \\
1\end{array}$ & $\begin{array}{l}\text { PSNR- } \\
2\end{array}$ & $\begin{array}{c}\text { PSNR- } \\
1\end{array}$ & $\begin{array}{c}\text { PSNR- } \\
2\end{array}$ & $\begin{array}{c}\text { PSNR- } \\
1\end{array}$ & $\begin{array}{c}\text { PSNR- } \\
2\end{array}$ & $\begin{array}{c}\text { PSNR- } \\
1\end{array}$ & $\begin{array}{l}\text { PSNR- } \\
2\end{array}$ & $\begin{array}{c}\text { PSNR- } \\
1\end{array}$ & $\begin{array}{c}\text { PSNR- } \\
2\end{array}$ & $\begin{array}{c}\text { PSNR- } \\
1\end{array}$ & $\begin{array}{c}\text { PSNR- } \\
2\end{array}$ \\
\hline 50 & 42.48 & 42.92 & 42.14 & 42.98 & 43.1 & 43.44 & 42.5 & 43.52 & 40.4 & 40.64 & 40.18 & 40.72 \\
\hline 20 & 53.38 & 53.70 & 53.22 & 53.6 & 53.98 & 54.00 & 53.78 & 54.18 & 51.6 & 51.72 & 51.5 & 51.66 \\
\hline 10 & 62.4 & 62.62 & 62.28 & 62.5 & 63.28 & 63.32 & 63.08 & 63.34 & 61.02 & 61.12 & 60.92 & 61.02 \\
\hline
\end{tabular}

Table.3: Comparison of PSNR-1 \& PSNR-2 values for RGB color images

\begin{tabular}{|c|c|c|c|c|c|c|c|c|c|c|c|c|}
\hline \multirow{3}{*}{$\begin{array}{l}\text { Noise } \\
\text { level }\end{array}$} & \multicolumn{4}{|c|}{ Barbara.tif } & \multicolumn{4}{|c|}{ House.tif } & \multicolumn{4}{|c|}{ Parrot.tif } \\
\hline & \multicolumn{2}{|c|}{ Normal } & \multicolumn{2}{|c|}{ Fast } & \multicolumn{2}{|c|}{ Normal } & \multicolumn{2}{|c|}{ Fast } & \multicolumn{2}{|c|}{ Normal } & \multicolumn{2}{|c|}{ Fast } \\
\hline & $\begin{array}{c}\text { PSNR- } \\
1\end{array}$ & $\begin{array}{c}\text { PSNR- } \\
2\end{array}$ & $\begin{array}{c}\text { PSNR- } \\
1\end{array}$ & $\begin{array}{c}\text { PSNR- } \\
2\end{array}$ & $\begin{array}{c}\text { PSNR- } \\
1\end{array}$ & $\begin{array}{c}\text { PSNR- } \\
2\end{array}$ & $\begin{array}{c}\text { PSNR- } \\
1\end{array}$ & $\begin{array}{c}\text { PSNR- } \\
2\end{array}$ & $\begin{array}{c}\text { PSNR- } \\
1\end{array}$ & $\begin{array}{c}\text { PSNR- } \\
2\end{array}$ & $\begin{array}{c}\text { PSNR- } \\
1\end{array}$ & $\begin{array}{c}\text { PSNR- } \\
2\end{array}$ \\
\hline 50 & 41.92 & 44.44 & 41.5 & 44.12 & 45.92 & 49.36 & 44.12 & 48.06 & 43.74 & 46.68 & 42.76 & 46.00 \\
\hline 20 & 54.26 & 55.78 & 54.22 & 55.76 & 57.34 & 59.04 & 56.44 & 58.48 & 56.26 & 58.28 & 55.94 & 58,06 \\
\hline 10 & 63.8 & 64.74 & 63.8 & 64.72 & 65.54 & 66.38 & 65.12 & 66.14 & 65.88 & 67.26 & 65.76 & 67.18 \\
\hline
\end{tabular}

\section{Original Image}

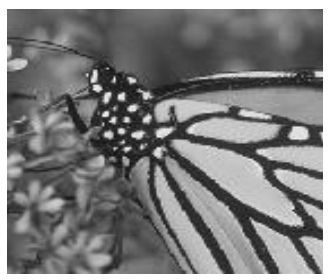

Noisy Image

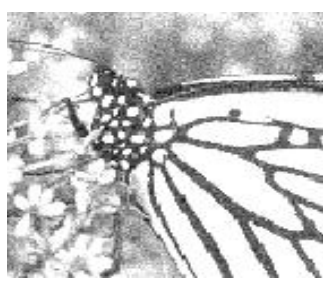

Image after DWT

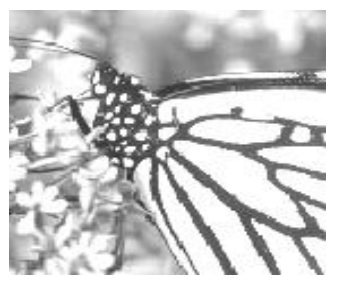

Denoised Image

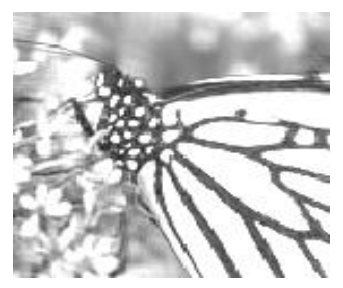

FIG.2 Results for noise level $=20$ (Fast mode $)$ 

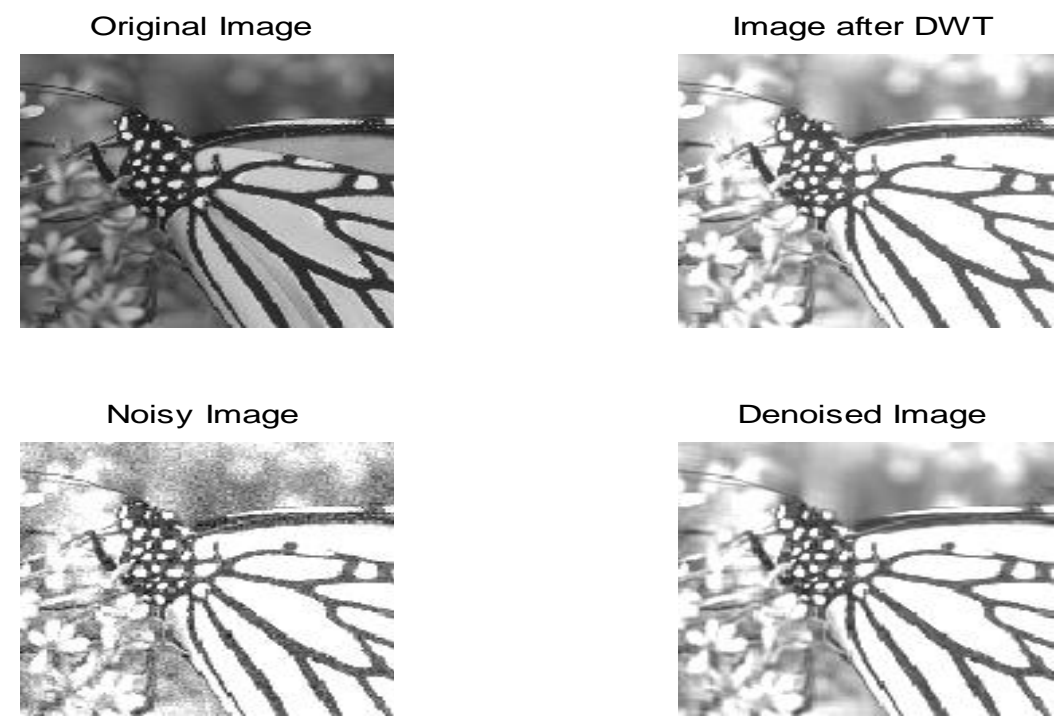

FIG.3 Results for noise level $=20$ (Normal mode)

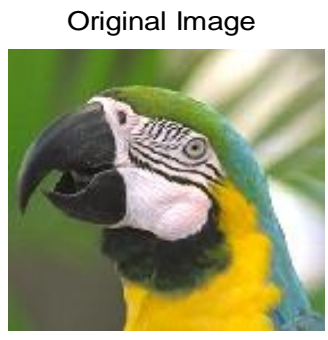

Image after DWT
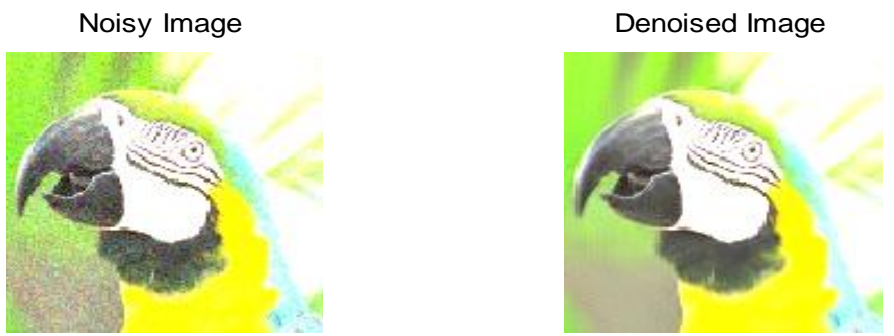

FIG.4 Results for noise level $=20($ Fast mode $)$

\section{CONCLUSION}

This paper introduced a simple and new algorithm to enhance the contrast of an image using Discrete wavelet transform. The paper shows the strengths of Adaptive principal component analysis by employing it to image denoising. In future IA-PCA algorithm can be use for video denoising and also denoising process can be fasten by adding compression technique.

\section{REFERENCES}

[1] Weisheng Donga, b, Lei Zhangb, "Image deblurring and Super-resolution by Adaptive Sparse Domain Selection and Adaptive Regularization", IEEE Transactions on Image Processing, vol. 20, no. 7, pp. 1838-1857, 2011.

[2] G. Y. Chen and B. Kegl, "Image denoising with complex ridgelets Pattern Recognition", vol. 40, pp. 78- 585, 2007. 
[3] J. L. Starck, E. J. Candes, and D. L. Donoho, "The curvelet transform for image denoising," IEEE Transactions on Image Processing, vol. 11, no. 6, pp. 670-684, 2002.

[4] Xin Li, "Fine-Granularity and Spatially-Adaptive Regularization for Projection-Based Image Deblurring" IEEE TRANSACTIONS ON IMAGE PROCESSING, VOL. 20, NO. 4, APRIL 2011.

[5] Oleg V. Michailovich, "An Iterative Shrinkage Approach to Total-Variation Image Restoration," IEEE transactions on image processing, vol. 20, no. 5, may 2011.

[6] Miyoun Jung, Xavier Bresson, Tony F. Chan, and Luminita A. Vese, "Nonlocal Mumford-Shah Regularizers for Color Image Restoration", IEEE transactions on image processing, vol. 20, no. 6, June 2011.

[7] Weisheng Dong, Lei Zhang, Member, IEEE, Guangming Shi, Senior Member, IEEE, and Xiaolin Wu, "Image Deblurring and Super-Resolution by Adaptive Sparse Domain Selection and Adaptive Regularization," IEEE Transactions on image processing, vol. 20, no. 7, July 2011

[8] Chao Wang, Lifeng Sun, Peng Cui, Jianwei Zhang, and Shiqiang Yang, "Analyzing Image Deblurring Through Three Paradigms", IEEE Transactions on image processing, vol. 21, no. 1, January 2012

[9] M. Prabukumar and J. Cristopher Clement. "Compressed Domain Contrast and Brightness Improvement Algorithm for Color Image through Contrast Measuring and Mapping of DWTCoefficients" International Journal of Multimedia and Ubiquitous Engineering Vol. 8, No. 1, January, 2013.

[10] [10].Hari Om, Mantosh Biswas. "An Improved Image Denoising Method Based on Wavelet Thresholding". Journal of Signal and Information Processing, 2012, 3, 109-116. doi:10.4236/jsip.2012.31014 Published Online February 2012 (http://www.SciRP.org/journal/jsip)

[11] Qingfu Zhang, Hujun Yin and Nigel M Allinson. "A Simplified ICA Based Denoising Method". 0-7695-0619$4 / 00 \$ 10.0002000$ IEEE
[12] Y. Murali Mohan Babu,Dr. M.V. Subramanyam, Dr. M.N. Giri Prasad. "PCA based image denoising. Signal \& Image Processing". An International Journal (SIPIJ) Vol.3, No.2, April 2012 DOI : 10.5121/sipij.2012.3218 236

[13] Taeg Sang Cho, C. Lawrence Zitnick, "Image Restoration by Matching Gradient Distributions," IEEE Transactions on pattern analysis and machine intelligence, vol. 34, no. 4, April 2012

[14] R.C.Gonzalez, R.E.Woods, Digital Image Processing,second Ed. Prentice- Hall, Englewood Cliffs, NJ, 2002

[15] M.Welk, D.Theis, J.Weickert, "Variational deburring of images with uncertain and spatially variant blurs. Pattern recognition," Mathematical image analysis group, Sarland University, Germany, vol.8, pp.33-40, 2005.

[16] Hui Ji and Kang Wang, "Robust Image Deblurring With an Inaccurate Blur Kernel", IEEE Transactions on Image processing, vol. 21, no. 4, April 2012

[17] C.Tomasi, R.Manduchi, Bilateral filtering for gray and color images, in: Proceedings of the 1998 IEEE International Conference on Computer Vision, Bombay, India, 1998, pp.839-846

[18] D.Barash, A fundamental relationship between bilateral filtering, adaptive smoothing, and then on linear diffusion equation, IEEE Transaction on Pattern Analysis and Machine Intelligence24 (6) (2002) 844-847.

[19] D.D.Muresan, T.W.Parks, "Adaptive principal components and image denoising", Proceedings of the 2003 International Conference on ImageProcessing, 14 17September,vol.1,2003,pp.I101-I104.

[20] Lei Zhang,Rastislav Lukac, Xiaolin Wu, and David Zhang, "PCA-Based Spatially Adaptive Denoising of CFAImages for Single-Sensor Digital Cameras" IEEE TRANSACTIONS ON IMAGE PROCESSING, VOL. 18 NO.4,APRIL2009797 